\title{
DETERMINACIÓN DE TASAS DE CRECIMIENTO DE TRÁFICO PROMEDIO DIARIO ANUAL EN NICARAGUA A PARTIR DE DATOS MACROECONÓMICOS
}

\section{DETERMINATION OF THE AVERAGE DAILY ANNUAL TRAFFIC GROWTH RATES IN NICARAGUA BASED ON MACROECONOMIC DATA}

\author{
Sergio Junior Navarro Hudiel ${ }^{1}$ \\ José Luis Acuña Mendoza ${ }^{2}$
}

(Recibido/received: 25-octubre-2021; aceptado/accepted: 4-diciembre-2021)

RESUMEN: La tasa de crecimiento vehicular es la medida estadística de mayor relevancia para determinar el comportamiento del tráfico futuro, utilizado en el diseño geométrico y espesores estructurales de pavimento. Debido a que las variables indicadoras del crecimiento vehicular en Nicaragua no son sectorizadas se debe normalizar los criterios a emplear en la selección de dichas varíales, así como la metodología para determinar la tasa de crecimiento de diseño vial y su posterior revisión. En este artículo se muestra el análisis de un criterio adicional para la determinación de tasas de crecimiento de forma generalizada es decir al uso de una única tasa para toda la composición vehicular, la cual correspondería a un valor de tráfico normal, proponiendo criterios para su uso y aceptación.

PALABRAS CLAVE: Proyecciones de tráfico; tasas de crecimiento; indicadores macroeconómicos

ABSTRACT: The vehicle growth rate is the most relevant statistical measure to determine the behavior of future traffic, used in the geometric design and structural pavement thicknesses. Due to the fact that the indicator variables of vehicle growth in Nicaragua are not sectorized, the criteria to be used in the selection of said variables should be standardized, as well as the methodology to determine the growth rate of road design and its subsequent revision. This article shows the analysis of an additional criterion for the determination of growth rates in a generalized way, that is, the use of a single rate for the entire vehicle composition, which would correspond to a normal traffic value, proposing criteria for its use. and acceptance

\footnotetext{
1 Universidad Nacional de Ingeniería Estelí, Nicaragua. Sergio.Navarro@norte.uni.edu.ni, Orcid: https://orcid.org/00000002-7001-5860.

2 Universidad Nacional de Ingeniería Managua, Nicaragua.Jose.Acuna@norte.uni.edu.ni, Orcid https://orcid.org/00000003-4475-3998
} 
KEYWORDS: Traffic projections; growth rates; macroeconomic indicators

\section{INTRODUCCIÓN}

Como refieren Ashhad, Cabrera y Roa (2021), Cal y Mayor (2018) y Sun (2016) el Tráfico Promedio Diario Anual es una de las variables que definen tanto las características geométricas de un tramo así como la estructura de pavimento, lo cual denota la importancia del cálculo adecuado de las tasas de crecimiento basado en comportamiento actual así como registros históricos. Es de vital importancia la definición del tránsito actual y futuro que permita la cuantificación de cargas impuestas del tráfico vehiculas que permita el correcto diseño tanto de la estructura de pavimentos, así como el diseño geométrico en correspondencia con las normas que rigen en cada país.

Como expresan Navarro y Bustamante Arteaga (2021) en referencia a MTI (2021), MTI (2020), MTI (2018) y MTI (2016) los Anuarios de aforo de tráfico publicados por el Ministerio de Transporte son un referente del comportamiento de los volúmenes de tráfico en las carreteras del país, para las estaciones principales.

Para Nicaragua por los altos costos incurridos no se realizan conteos clasificados de los volúmenes de tránsito durante todo el año sino divididos en tres cuatrimestres (MTI, 2019, p.9). Se considera que el Tránsito Promedio Diario Anual será "el volumen vehicular que representa el promedio de todos los volúmenes diarios en un año" (SCT, 2016, p.11).

En Nicaragua acorde a Anuario de Aforo Tráfico 2018, publicado por el Ministerio de Transporte e Infraestructura (MTI, 2021) referido por Navarro y Bustamante (2021), a través de la Oficina de diagnóstico, evaluación de pavimentos y puentes, desde el año 2010 en Nicaragua se aplican vectores de correspondencia para identificar las características, volúmenes de tráfico de las estaciones, perfil de variación en el flujo vehicular y el grado de relación entre las diferentes estaciones de la red vial.

\section{METODOLOGÍA}

Acorde a Hernández, Fernández y Baptista (2014) esta investigación es de tipo descriptiva con un enfoque cuantitativo. Para la descripción de las características y fuentes de información estadística de conteos clasificados se realizó una revisión documental, así como otros recursos encontrados en la internet de los últimos cinco años. Para el análisis y proyección de datos de tráfico clasificado así como el cálculo de tasas de crecimiento se hizo uso de la metodología propuestas en anuarios de tráfico del Ministerio de Transporte e Infraestructura de Nicaragua, aplicando de cálculo de tasas de crecimiento con valores extremos así como media geométrica para los valores positivos, de igual manera se aplicó regresión lineal por mínimos cuadrados propuestos por MTI (2019), Barraza Ortiz, Domínguez Solano y Herrera Acosta (2019), SCT (2016) aplicados al tramo de carretera ubicado en San Sebastián de Yalí - El Tigre como caso de estudio. 


\section{RESULTADOS Y DISCUSIÓN}

Para este artículo se retoman los resultados descritos en artículo "Cálculo de tasa de crecimiento de tránsito para proyecciones de tráfico promedio diario anual en Nicaragua" elaborado por Navarro y Bustamante (2021) en el cual se proponen correlaciones entre TPDA-PIB. TPDA Población y registros históricos de los datos de tráfico estadísticos de los anuarios publicados por el MTI, los dos principales resultados indican que:

- A partir de Datos históricos de TPDA disponibles para estación sumaria la tasa interanual de crecimiento entre los años 2015-2018 fue de $4.42 \%, 6.35 \%$ para los rangos promedios de la estación de mayor cobertura o tasa general de crecimiento para todos los vehículos.

- Bajo metodología propuesta la tasa de crecimiento para vehículos livianos fue de $6.35 \%$, tasa de crecimiento para transporte de carga $3.5 \%$ y para transporte de pasajeros $4.9 \%$.

Cabe señalar que en Nicaragua no existe una metodología definida para definir las tasas de proyección, sin embargo acorde al Manual para la Revisión de Estudios de Tránsito, elaborado con la colaboración y financiamiento del Real Gobierno de Dinamarca por medio del Programa de Apoyo al Sector Transporte - PAST-DANIDA y con el decisivo apoyo y dirección del Ministerio de Transporte e Infraestructura por medio de la División General de Planificación (DGP) en el año 2008, para determinar las proyecciones de tránsito se emplean varios procedimientos, "que van desde los más complejos y sofisticados a base de modelos econométricos hasta los que se caracterizan por su extrema simplicidad en el cálculo o dependen solamente del buen juicio y criterio del diseñador" continúan su planteamiento refiriendo:

Entre ellos, dos procedimientos son universalmente aceptados, aunque cabe señalar que a mayor incertidumbre en las estimaciones a futuro, mayor será la conveniencia de complementar los estudios con un análisis de sensibilidad para prever situaciones extremas en las perspectivas a largo plazo. El primero se fundamenta en el análisis de las tendencias históricas del comportamiento del tránsito, conocidas mediante registros de los volúmenes durante un período mínimo de diez años de duración, para desprender de ellas las hipótesis de crecimiento más probable del tránsito durante los años venideros, en sus diferentes componentes de la corriente vehicular. El segundo método reconoce que los pronósticos de tránsito guardan estrecha relación con indicadores de las múltiples actividades humanas, cuyos patrones relacionados con la movilidad, se consideran invariables en el período de diseño de las obras viales, a menos que se conozcan de antemano factores que pueden influir en su futuro comportamiento. Bajo este criterio general se busca establecer relaciones razonables de tipo estadístico entre el comportamiento del tránsito (variable dependiente) y el de otros conocidos indicadores socioeconómicos nacionales o locales, que tienen incidencia en el transporte automotor, como los registros de consumo de combustible (gasolina, diésel o ambos) en el transporte, la tenencia de vehículos, el comportamiento del Producto Interno Bruto, el crecimiento de la población económicamente activa, (variables independientes), que permitan obtener 
proyecciones aceptables acerca de los futuros volúmenes de tránsito por las carreteras" (MTI, 2008, pp. 90-91).

La propuesta de determinación de estas tasas es más simplista y práctica ajustado a variables socioeconómica centradas en tres variables referida al Producto Interno Bruto, Población, así como Consumo de combustible (Diesel y gasolina). Este planteamiento está acorde a las relaciones establecidas entre el crecimiento de PIB y el transporte, ya estudiado y evaluado por autores como Díaz (2020), Jordá y Lopez (2020), OECD ( 2019), Blass, Chavarría y Elisabeth (2019), López y Pardo (2019), Rendón, Hernández y Del Rio, 2019) Berrones, Canos, Sánchez y Martínez (2018), OCDE (2018), Machado (2017), Rodríguez, Mejía y Zapata (2015), Zamora y Pedraza (2013) y Sanabria (2008),

Se propone incorporar el análisis de variación de consumo de combustible, asociados al transporte. Como refiere el (MINEM, 2017, p.4) "las gasolinas y el diésel continúan siendo los más demandados por el sector transporte público y privado" siendo considerados asociados al crecimiento económico. Este comportamiento continua siendo reportado en las estadísticas nacionales, "los productos de mayor consumo nacional siguen siendo el Diésel, Fuel Oil y las Gasolinas con una participación del $35.01 \%$, 24.20\% y $23.64 \%$ respectivamente, acumulando conjuntamente el $82.85 \%$ de la demanda nacional de hidrocarburos MINEM (2020. p.4). Los datos de consumo histórico de gasolina y diésel mostrados en tabla 1.

Tabla 1. Consumo de hidrocarburos Nicaragua en miles de barriles

\begin{tabular}{|l|c|c|c|}
\hline Año & $\begin{array}{c}\text { Consumo } \\
\text { de Diesel }\end{array}$ & $\begin{array}{c}\text { Consumo de } \\
\text { Gasolina y } \\
\text { Diesel }\end{array}$ & $\begin{array}{c}\text { Tasa de crecimiento } \\
\text { Interanual }\end{array}$ \\
\hline 1994 & & & \\
\hline 1995 & $2,108.10$ & $3,041.80$ & $6.35 \%$ \\
\hline 1996 & $2,277.40$ & $3,234.90$ & $-1.62 \%$ \\
\hline 1997 & $2,608.80$ & $3,182.60$ & $13.00 \%$ \\
\hline 1998 & $3,156.50$ & $3,596.30$ & $19.35 \%$ \\
\hline 1999 & $2,978.70$ & $4,292.10$ & $-1.30 \%$ \\
\hline 2000 & $2,833.90$ & $4,236.40$ & $-1.92 \%$ \\
\hline 2001 & $2,863.70$ & $4,266.50$ & $2.68 \%$ \\
\hline 2002 & $2,769.10$ & $4,245.80$ & $-0.49 \%$ \\
\hline 2003 & $2,846.80$ & $4,357.20$ & $2.62 \%$ \\
\hline 2004 & $3,007.40$ & $4,571.30$ & $4.91 \%$ \\
\hline 2005 & $2,971.70$ & $4,553.40$ & $-0.39 \%$ \\
\hline 2006 & $3,079.70$ & $4,702.60$ & $3.28 \%$ \\
\hline 2007 & $3,505.70$ & $5,221.60$ & $11.04 \%$ \\
\hline 2008 & $3,153.10$ & $4,859.00$ & $-6.94 \%$ \\
\hline 2009 & $3,097.60$ & $4,918.10$ & $1.22 \%$ \\
\hline
\end{tabular}




\begin{tabular}{|l|c|c|c|}
\hline Año & $\begin{array}{c}\text { Consumo } \\
\text { de Diesel }\end{array}$ & $\begin{array}{c}\text { Consumo de } \\
\text { Gasolina y } \\
\text { Diesel }\end{array}$ & $\begin{array}{c}\text { Tasa de crecimiento } \\
\text { Interanual }\end{array}$ \\
\hline 2010 & $3,282.40$ & $5,143.20$ & $4.58 \%$ \\
\hline 2011 & $3,474.00$ & $5,388.00$ & $4.76 \%$ \\
\hline 2012 & $3,560.80$ & $5,615.90$ & $4.23 \%$ \\
\hline 2013 & $3,656.10$ & $5,788.50$ & $3.07 \%$ \\
\hline 2014 & $3,853.60$ & $6,127.10$ & $5.85 \%$ \\
\hline 2015 & $4,232.10$ & $6,813.70$ & $11.21 \%$ \\
\hline 2016 & $4,441.50$ & $7,287.50$ & $6.95 \%$ \\
\hline 2017 & $3,091.70$ & $5,049.30$ & $-30.71 \%$ \\
\hline
\end{tabular}

Fuente: Banco Central de Nicaragua, Cuadros 4.v.4.17.2 y 4.v.4.17.4 - Consumo de hidrocarburos. Dispuestos en https://www.ben.gob.ni/base-de-datos-estad\%C3\%ADsticos

Incluyendo todos los registros la tasa de crecimiento promedio es de $2.68 \%$. Excluyendo los datos de decrecimiento, el valor de tasa de crecimiento promedio es de $6.57 \%$. Este dato es un referente general para el crecimiento de consumo, asociado al transporte y por tanto representa un rango muy cercano al de las tasas de crecimiento reportado en estaciones sumarias.

Basado en regresión lineal como modelo predictivo adoptado por autores como Brenes (2018), Carrasquilla et al., (2016), Batanero, Gea, López y Arteaga (2017) Dagnino (2014) y acorde a Blass et al., (2019, p. 14) para elaborar las rectas de regresión y determinar tasas de crecimiento acorde a relaciones y ecuaciones generadas, se aplica el logaritmo neperiano a los datos de consumo de hidrocarburos, producto interno Bruto y Población, con el objetivo de reducir el rango de variación de variables y la sensibilidad a datos atípicos, los cuales al evaluar la pendiente de la recta como valor de tasas de crecimiento. Los datos a ser modelados se muestran en tabla 2.

Tabla 2. Indicadores de crecimiento del tránsito vehicular

\begin{tabular}{|c|c|c|c|}
\hline Año & $\begin{array}{c}\text { PIB a } \\
\text { precios } \\
\text { constantes } \\
\text { en millones } \\
\text { de } \\
\text { córdobas } \\
1 /\end{array}$ & $\begin{array}{c}\text { Población } \\
\text { (miles de } \\
\text { habitantes)2/ }\end{array}$ & $\begin{array}{c}\text { Consumo } \\
\text { de gasolina } \\
\text { y Diésel en } \\
\text { miles de } \\
\text { barriles }\end{array}$ \\
\hline 2,006 & 118,838 & 5,523 & $4,702.60$ \\
\hline 2,007 & 124,870 & 5,596 & $5,221.60$ \\
\hline 2,008 & 129,161 & 5,669 & $4,859.00$ \\
\hline 2,009 & 124,908 & 5,742 & $4,918.10$ \\
\hline 2,010 & 130,416 & 5,816 & $5,143.20$ \\
\hline 2,011 & 138,654 & 5,997 & $5,388.00$ \\
\hline 2,012 & 147,661 & 6,071 & $5,615.90$ \\
\hline 2,013 & 154,937 & 6,134 & $5,788.50$ \\
\hline
\end{tabular}




\begin{tabular}{|c|c|c|c|}
\hline Año & $\begin{array}{c}\text { PIB a } \\
\text { precios } \\
\text { constantes } \\
\text { en millones } \\
\text { de } \\
\text { córdobas } \\
1 /\end{array}$ & $\begin{array}{c}\text { Población } \\
\text { (miles de } \\
\text { habitantes)2/ }\end{array}$ & $\begin{array}{c}\text { Consumo } \\
\text { de gasolina } \\
\text { y Diésel en } \\
\text { miles de } \\
\text { barriles }\end{array}$ \\
\hline 2,014 & 162,351 & 6,198 & $6,127.10$ \\
\hline 2,015 & 170,132 & 6,263 & $6,813.70$ \\
\hline 2,016 & 177,895 & 6,328 & $7,287.50$ \\
\hline 2,017 & 186,134 & 6,394 & $5,049.30$ \\
\hline 2,018 & 179,873 & 6,460 & \\
\hline 2,019 & 173,264 & 6,528 & \\
\hline 2,020 & 169,838 & 6,596 & \\
\hline
\end{tabular}

1/ : : PIB para el período 2006-2020 (año de referencia 2006)

2/ : Estimaciones de población para cada año, en base al censo de población 2005 y de ENDESA 2006-2007, y revisión 2012

Fuente: Banco Central de Nicaragua, Principales Indicados. Dispuesto en https://www.bcn.gob.ni/cuadros-de-anuario-de-estadisticas-macroeconomicas-1960-2020

Los datos mostrados en tabla 3 , con los que se generaron ecuaciones basadas en mínimos cuadrados y cuya pendiente será considerada como tasa de crecimiento interanual se muestra en las figuras 2,3 y 4 .

Tabla 3. LN de los indicadores de crecimiento del tránsito vehicular

\begin{tabular}{|r|c|c|c|}
\hline Año & Ln PIB & Ln Población & Ln Consumo Hidrocarburos \\
\hline 2,006 & 11.6855165 & 8.61667647 & 8.455870826 \\
\hline 2,007 & 11.73502848 & 8.629807336 & 8.560559147 \\
\hline 2,008 & 11.76881497 & 8.642768014 & 8.488587934 \\
\hline 2,009 & 11.73533275 & 8.655562861 & 8.500677556 \\
\hline 2,010 & 11.77848462 & 8.668368019 & 8.545430733 \\
\hline 2,011 & 11.8397369 & 8.699014623 & 8.591929538 \\
\hline 2,012 & 11.90267438 & 8.711278615 & 8.633357139 \\
\hline 2,013 & 11.95077386 & 8.721602345 & 8.66362847 \\
\hline 2,014 & 11.99751594 & 8.731981938 & 8.720476834 \\
\hline 2,015 & 12.04432989 & 8.742414583 & 8.82669057 \\
\hline 2,016 & 12.08894877 & 8.752739509 & 8.893915831 \\
\hline 2,017 & 12.13422212 & 8.76311533 & 8.527004899 \\
\hline 2,018 & 12.10000633 & 8.773384597 & \\
\hline 2,019 & 12.06257172 & 8.783855897 & \\
\hline 2,020 & 12.04260032 & 8.794218684 & \\
\hline
\end{tabular}

Fuente: Elaborada a partir de datos publicados por Banco Central de Nicaragua 


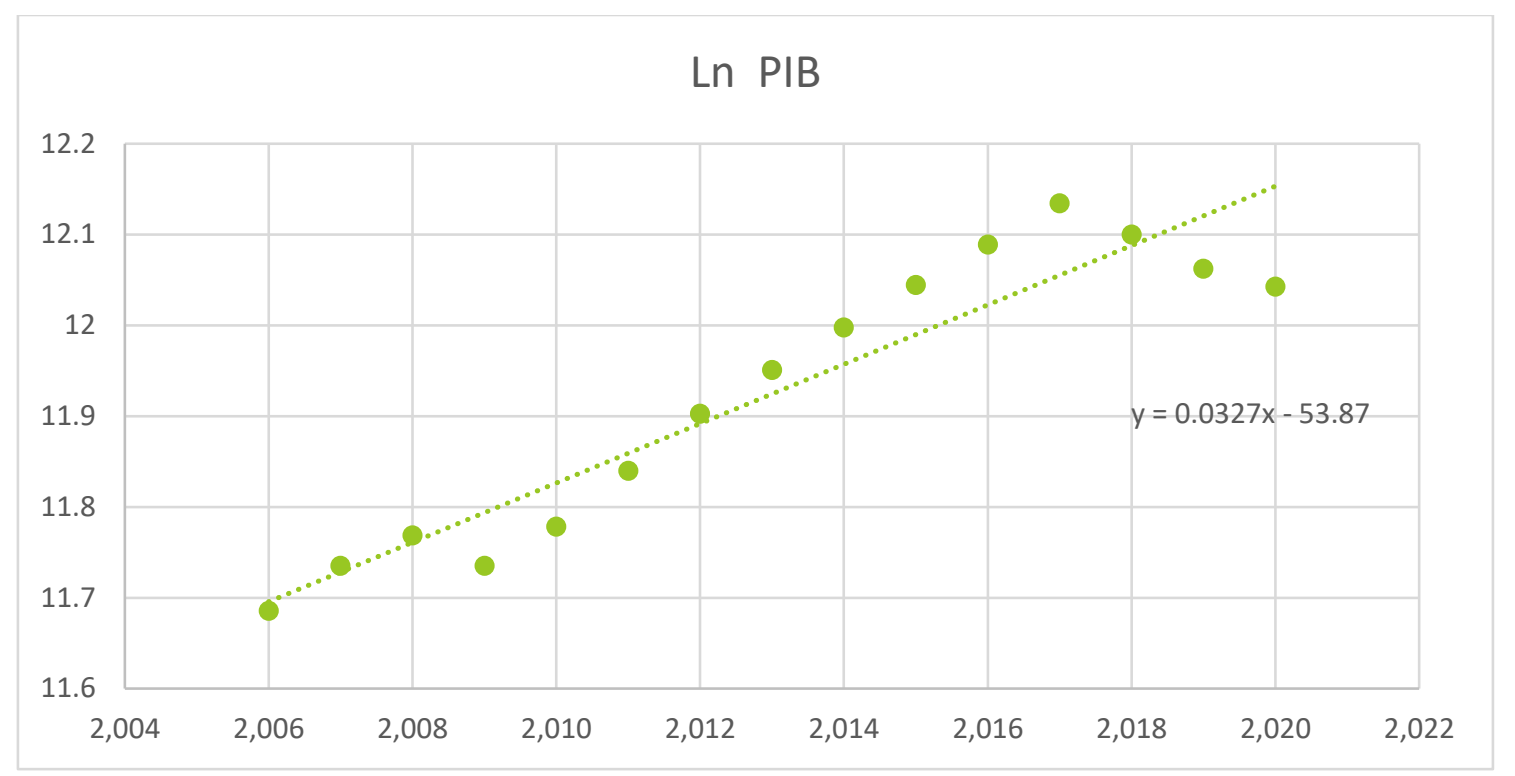

Figura 1. Ln Producto Interno Bruto

La Tasa de crecimiento de población, es de $3.27 \%$, misma que aproxima a los rangos establecidos como mínimo de $2.65 \%$, en promedio incluyendo todos los datos reportados en periodo 2006-2020, y $4.94 \%$ considerando solamente las tasas de crecimiento interanuales 0 positivas, registradas y reportada por el Banco Central de Nicaragua.

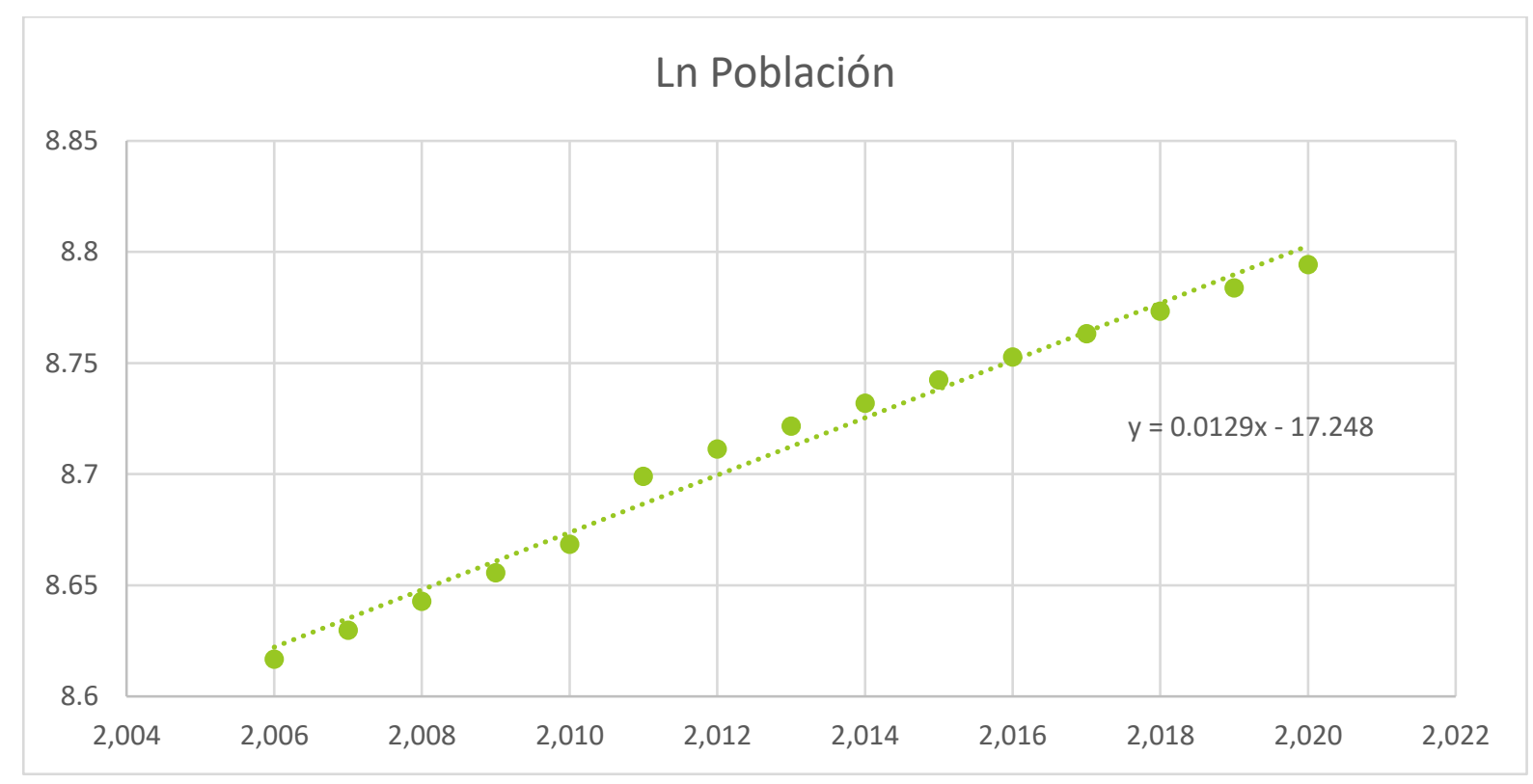

Figura 2. Ln Población

Tasa de crecimiento de población, es de $1.29 \%$, misma que coincide en cuanto a tasa promedio de $1.28 \%$ reportada por el Banco Central de Nicaragua. 


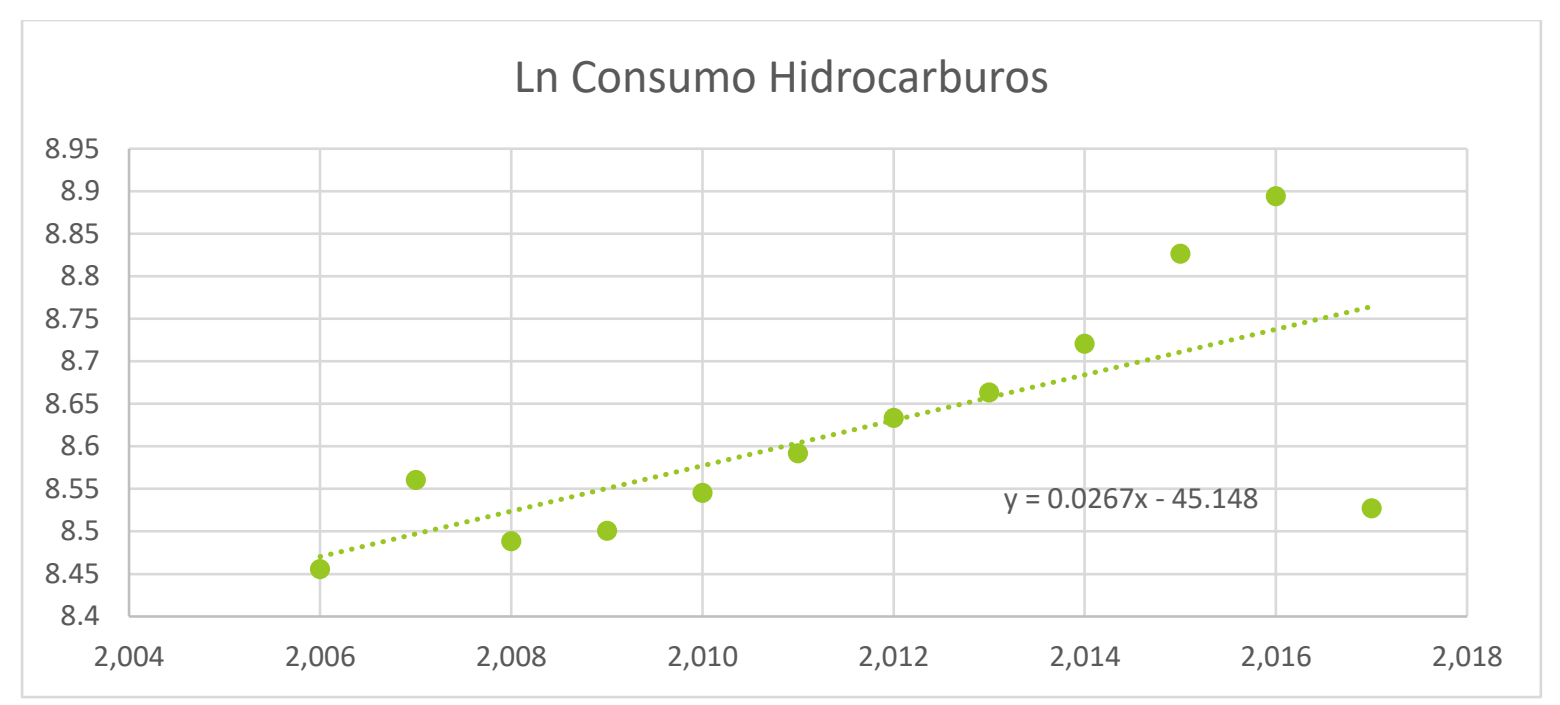

Figura 3. Ln Consumo hidrocarburos

Tasa de crecimiento de consumo de Hidrocarburos, es de $2.67 \%$, misma que aproxima a los rangos establecidos como mínimo de $2.68 \%$, en promedio incluyendo todos los datos reportados en periodo 2006-2017, y $6.57 \%$ considerando solamente las tasas de crecimiento interanuales o positivas, registradas y reportada por el Banco Central de Nicaragua.

Acorde a Blass et al., (2019) puede hacerse la proyección del tránsito utilizando el promedio de las tasas del PIB, Consumo de Combustible y Población. De esta manera la tasa de crecimiento, que podría ser asociada al tráfico normal será el promedio equivalente a $2.41 \%$ del promedio de los datos $3.27 \%, 1.29$, y $2.67 \%$ correspondientes a la pendiente de las ecuaciones basadas en los mínimos cuadrados comprendidos en el periodo de 2006-2017.

En correspondencia con las tasas de crecimiento planteadas por Navarro y Bustamante (2021) la tasa de crecimiento para vehículos livianos fue de $6.35 \%$, tasa de crecimiento para transporte de carga $3.5 \%$ y para transporte de pasajeros $4.9 \%$, lo cuales son relativamente altas en función de la nueva tasa general o del tráfico normal de $2.41 \%$.

Retomando lo planteado MTC (2013) por en la que la tasa anual de crecimiento del tránsito esta previamente establecida en su mecanismo de cálculo, Las Normas Peruanas plantean:

Se define en correlación con la dinámica de crecimiento socio-económico. Normalmente se asocia la tasa de crecimiento del tránsito de vehículos de pasajeros con la tasa anual de crecimiento poblacional; y la tasa de crecimiento del tránsito de vehículos de carga con la tasa anual del crecimiento de la economía expresada como el Producto Bruto Interno (PBI). Normalmente las tasas de crecimiento del tráfico varían entre $2 \%$ y $6 \%$.

Dado las diferencias entre la selección de tasas de crecimiento, se plantea usar las que sean ajustadas al comportamiento real del tráfico acorde a registros históricos. Los indicadores generales basados en datos macroeconómicos no son los suficientemente detallados por regiones o zonas, que asocien a actividades de la zona y capacidad productiva actual y futura, 
por lo cual serán referente para la selección de tasas. Se propone usar tasas diferenciadas por tipo de vehículo y encontradas por las correlaciones acorde a Navarro y Bustamante (2021). Deben ser usadas las estaciones sumarias o de control más cercanas encontradas a las zonas y tomar de referencia las de dependencia, debido a que las actividades económicas, población y estrados difieren por ubicación geográfica.

Para concluir la selección de tasas, es mejor valorar el comportamiento de estaciones más cercanas al tramo en estudio. Así en general para la estación en análisis, estación 3503 de corta duración número 218, código NIC-35D, los datos históricos reportan crecimiento de hasta $7.32 \%$, se muestran en tabla 4.

Tabla 4. Composición vehicular de la estación de corta duración 3503

\begin{tabular}{|c|c|c|c|c|c|c|c|c|}
\hline Año & Autos & Jeep & Cmta. & $\begin{array}{c}\text { MnBus } \\
15-30 \text { s. }\end{array}$ & Bus & $\begin{array}{c}\text { Total } \\
\text { Livianos }\end{array}$ & $\begin{array}{c}\text { Liv. } \\
2-5 \text { Ton }\end{array}$ & $\begin{array}{c}\text { C2 } \\
5+\text { Ton }\end{array}$ \\
\hline 2018 & 4 & 2 & 200 & 2 & 17 & 19 & 14 & 12 \\
\hline 2015 & 3 & 2 & 162 & 2 & 14 & 16 & 11 & 10 \\
\hline 2009 & & 10 & 103 & & 19 & 19 & 2 & 18 \\
\hline 2006 & & 6 & 69 & & 17 & 17 & 6 & 15 \\
\hline 2002 & 1 & 12 & 80 & & 15 & 15 & 16 & 15 \\
\hline 1999 & & 10 & 72 & & 22 & 22 & 6 & 9 \\
\hline
\end{tabular}

Fuente: $\operatorname{MTI}(2019$, p. 176)

Agrupando por categorías, excluyendo las motos por su capacidad de carga se generaron los volúmenes de tráfico de tabla 5 .

Tabla 5. Composición vehicular por categoría

\begin{tabular}{|c|c|c|c|}
\hline Año & $\begin{array}{c}\text { Total } \\
\text { Livianos }\end{array}$ & $\begin{array}{c}\text { Total } \\
\text { Pasajeros }\end{array}$ & $\begin{array}{c}\text { Total } \\
\text { Carga }\end{array}$ \\
\hline 2018 & 206 & 19 & 26 \\
\hline 2015 & 167 & 16 & 21 \\
\hline 2009 & 113 & 19 & 20 \\
\hline 2006 & 75 & 17 & 21 \\
\hline 2002 & 93 & 15 & 31 \\
\hline 1999 & 82 & 22 & 15 \\
\hline
\end{tabular}

Aplicando las ecuaciones propuestas por MTI (2020, pp. 14-15) y ya desarrolladas por Navarro y Bustamante (2021) se determina tasas de crecimiento entre periodos y medias geométricas cuyos resultados se muestran en tabla 6. 
Tabla 6. Tasa de crecimiento inter anual y media geométrica.

\begin{tabular}{|c|c|c|c|c|c|c|}
\hline Año & $\begin{array}{c}\text { Total } \\
\text { Livianos }\end{array}$ & $\begin{array}{c}\text { Tasa Crecimiento } \\
\text { entre años entre } \\
\text { periodos } \\
\text { registrados }\end{array}$ & $\begin{array}{c}\text { Total } \\
\text { Pasajeros }\end{array}$ & $\begin{array}{c}\text { Tasa } \\
\text { Crecimiento } \\
\text { entre } \\
\text { periodos } \\
\text { registrados }\end{array}$ & $\begin{array}{c}\text { Total } \\
\text { Carga }\end{array}$ & $\begin{array}{c}\text { Tasa } \\
\text { Crecimiento } \\
\text { entre } \\
\text { periodos } \\
\text { registrados }\end{array}$ \\
\hline 2018 & 206 & & 19 & & 26 & \\
\hline 2015 & 167 & $7.247 \%$ & 16 & $5.896 \%$ & 21 & $7.379 \%$ \\
\hline 2009 & 113 & $6.727 \%$ & 19 & $-2.824 \%$ & 20 & $0.816 \%$ \\
\hline 2006 & 75 & $14.641 \%$ & 17 & $3.777 \%$ & 21 & $-1.613 \%$ \\
\hline 2002 & 93 & $-5.236 \%$ & 15 & $3.179 \%$ & 31 & $-9.278 \%$ \\
\hline 1999 & 82 & $4.285 \%$ & 22 & $-11.985 \%$ & 15 & $27.377 \%$ \\
\hline
\end{tabular}

\begin{tabular}{|l|l|l|l|l|l|}
\hline Media Geométrica & $7.437 \%$ & & $4.137 \%$ & & $5.484 \%$ \\
\hline
\end{tabular}

Las tasas de crecimiento por tipo de vehículo representan condiciones actuales de los corredores y deben ser las bases de cálculo para proyecciones futuras.

Es notorio que el incremento del flujo vehicular categorizado en livianos, pasajeros y carga aumentan de manera desigual por lo tanto aplicar una tasa de crecimiento generalizada para todas la categorías no explicaría de la mejor manera el comportamiento de cada una de ellas, considerando que los anuarios del MTI facilitan los datos observados en el tramo de estudio se debe elegir variables indicadoras de crecimiento que se ajusten al incremento por categoría como fue mencionado anteriormente.

Mientras no se tenga definido la metodología para evaluar tasas de crecimiento normalizadas, estas deben de ser determinadas a partir de los históricos del comportamiento de estaciones de conteo más cercanas, se recomienda el uso de tasas diferenciadas por categoría y emplear incrementos de 20-30\% que correspondan a tráfico generado y desarrollado, a la falta de datos, este último porcentaje representará un factor de seguridad para las estimaciones. El uso de las variables macroeconómicas, al igual que las tasas de crecimiento de las estaciones principales, representan un referente para su uso en estaciones de cobertura o sumarias. En tramo en estudio podrán ser empleadas las tasas de crecimiento para vehículos livianos de $6.35 \%$, transporte de carga $3.5 \%$ y transporte de pasajeros $4.9 \%$.

\section{CONCLUSIONES}

Los datos de referencia de consumo de Hidrocarburos podrán ser considerados como un referente como criterio para la selección de tasas de crecimiento vehicular estando en rangos de $2.68 \%$ siendo este el valor resultante del promedio al incluir todos los datos del indicador de crecimiento del tráfico antes mencionado y $6.57 \%$ el correspondiente al promedio excluyendo los 
decrecimientos, lo cual son valores aceptables en relación a los rangos de 3.36 - 6\% publicados en anuarios estadísticos de tráfico acorde al comportamiento de las principales estaciones de aforo en Nicaragua, así como crecimiento de PIB entre 2 y $6 \%$ indicados en anuarios estadísticos del banco central de Nicaragua. De igual manera podrán ser incluidos en análisis otros indicadores macroeconómicos publicados por el banco central.

Es necesario que se definan criterios normalizados para determinar las tasas de crecimiento de parque vehicular siendo los indicados macroeconómicos del país así los históricos registros de estaciones de mayor cobertura valores de referencia para los rangos de variación aceptables. En nuestro país podrá ser aceptada tasas de hasta $7.5 \%$, correspondientes a la media geométrica de los datos publicados por el Ministerio de Transporte e Infraestructura (MTI - Nicaragua) a través de los anuarios de tráfico elaborados por Oficina de Diagnóstico y Evaluación de Pavimentos y Puentes en la División de Administración Vial.

Basado en análisis de tendencias se sugiere, para este análisis, usar las tasas ya calculadas por correlaciones entre indicados macroeconómicos y PIB asociadas al comportamiento del tráfico reflejado en anuarios estadísticos equivalente a $6.35 \%$ para los livianos, $3.5 \%$ para carga y para transporte de pasajeros $4.9 \%$ las que de manera general resultan en $0.72 \%$ de diferencia de los resultados referidos en artículo base de Navarro y Bustamante (2021).

Por la falta de datos de aforo de tráfico continuos, se sugiere la incorporación de tasas de crecimiento adicionales al tráfico normal, en lo general con valores de alrededor del $20 \%$ para los valores de tráfico desarrollado y generado referidos por Cal y Mayor (2018). Los datos de crecimiento de estaciones de conteo, distintas a las principales de Nicaragua, deben de tomar una tasa de crecimiento de referencia que no exceda los registros históricos. Expertos en esta temática, podrán generar aportes a esta propuesta que abone a unificar criterios en la determinación de tasas de crecimiento.

\section{REFERENCIAS}

Ashhad, T., Cabrera, F. y Roa, O. (2021). Análisis del congestionamiento vehicular para el mejoramiento de vía principal en Guayaquil-Ecuador. Gaceta Técnica, 2, 1-14. Recuperado de https://www.redalyc.org/jatsRepo/5703/570363740001/html/index.html

Barraza, E., Domínguez, L. y Herrera, R. (2019). Cálculo del indice de capacidad de procesos usando media geométrica. Investigación e Innovación En Ingenierías, 7(2), 86-95. https://doi.org/10.17081/invinno.7.2.2851

Batanero, C., Gea, M., López, M. del M. y Arteaga, P. (2017). Análisis de los conceptos asociados a la correlación y regresión en los textos de bachillerato. Didacticae, (1), 60-76. https://doi.org/10.1344/did.2017.1.60-76

Berrones, L., Canos, P., Sánchez, D. y Martínez, J. (2018). Entregas tardías o incorrectas en el autotransporte de carga y su relación con las condiciones laborales de los choferes: Un modelo de regresión logística. Nova Scientia, 10(20), 413-441. https://doi.org/10.21640/ns.v10i20.1063 
Blass, A., Chavarría, Z. y Elisabeth, S. (2019). Diseño geométrico y de pavimento articulado, de $6.3 \mathrm{~km}$ de camino el retén-Santa Sna del municipio La Concepción, Masaya. Universidad Nacional de Ingeniería.

Brenes, H. (2018). Aplicación del análisis de regresión lineal simple para la estimación de los precios de las acciones de Facebook, Inc. REICE: Revista Electrónica de Investigación En Ciencias Económicas, 5(10), 133-155. https://doi.org/10.5377/reice.v5i10.5535

Cal y Mayor, R. C. J. (2018). Ingeniería de tránsito Fundamentos y aplicaciones (Novena edi). México: Alfaomega Grupo Editor.

Carrasquilla, A., Chacón, A., Núñez, K., Gómez, O., Valverde, J. y Guerrero, M. (2016). Regresión lineal simple y múltiple: aplicación en la predicción de variables naturales relacionadas con el crecimiento microalgal. Revista Tecnología En Marcha, 29(8), 33. https://doi.org/10.18845/tm.v29i8.2983

Dagnino, J. (2014). REGRESIÓN LINEAL. Revista Chilena de Anestesia, 43(2). https://doi.org/10.25237/revchilanestv43n02.14

Díaz, O. (2020). Contribución del transporte aéreo a la conectividad territorial. El caso de Colombia. EURE, 47(140). https://doi.org/10.7764/EURE.47.140.06

López, C. y Pardo, S. (2019). El transporte de carga terrestre en el comercio internacional. Análisis comparativo entre Bogotá, Colombia y Santa Cruz de la Sierra, Bolivia. Ensayos de Economía, 29(54), 89-114. https://doi.org/10.15446/ede.v29n54.75022

Machado, R. (2017). Crecimiento económico e infraestructura de transportes y comunicaciones en el Perú. Economía, 40(79), 9-46. https://doi.org/10.18800/economia.201701.001

MINEM. (2017). Anuario Estadísticas de Hidrocarburos 2016". (MINEM Ministerio de Energía y Minas, Ed.). Managua.

MINEM. (2020). Anuario estadístico de Hidrocarburos 2019. (M. de E. y M. MINEM, Ed.). Managua: Ministerio de Energía y Minas. Retrieved from http://www.mem.gob.ni/wpcontent/uploads/2021/03/Anuario-Estadistico-2019-sin-USGC.pdf

MTC, M. de T. y C. (2013). Manual de Carreteras "Suelos, Geología, Geotecnia y Pavimentos." (Ministerio de Transportes y Comunicaciones, Ed.). Lima.

MTI. (2008). Manual para la Revisión de Estudios de Tránsito. Managua: Programa de Apoyo al Sector Transporte - PAST-DANIDA.

MTI. (2016). Anuario de Aforo de Tráfico año 2015. Managua: Ministerio de Transporte e Infraestructura.

MTI. (2018). Anuario de Aforo de Tráfico año 2017. Managua: Ministerio de Transporte e Infraestructura.

MTI. (2019). Anuario de Aforo de Tráfico año 2018. Managua: Ministerio de Transporte e Infraestructura.

MTI. (2020). Anuario de aforos de tráfico año 2019. Managua: Ministerio de Transporte e Infraestructura. 
MTI. (2021). Anuarios de aforos de Tráfico 2020. Managua: Ministerio de Transporte e Infraestructura.

Navarro, S. y Bustamante, F. (2021). Cálculo de tasa de crecimiento de tránsito para proyecciones de tráfico promedio diario anual en Nicaragua. Revista Ciencia y Tecnología El Higo, 11(1), 43-68. https://doi.org/10.5377/elhigo.v11i1.11717

OCDE. (2018). Perspectivas económicas de América Latina 2019. (O. U. 2018, Ed.). Centro de Desarrollo de la Organización para la Cooperación y el Desarrollo Económicos - OECD. https://doi.org/10.1787/leo-2018-es

OECD. (2019). Perspectivas económicas de América Latina 2019. (C. E. para A. L. y el C. de las N. U. CEPAL, Ed.). Paris: OECD. https://doi.org/10.1787/g2g9ff1a-es

Rendón, J., Hernández, E. y Del Rio, H. (2019). Nueva generación de Modelos de transporte a través del uso de BIG DATA Caso San Salvador. Washington, D.C.: Transportation Research Board. https://doi.org/10.17226/25519

Rodríguez, M., Mejía, M. y Zapata, S. (2015). La causalidad entre el crecimiento económico y la expansión del transporte aéreo: un análisis empírico para Chile. Revista de Economía Del Rosario, 18(1), 127-144. https://doi.org/10.12804/rev.econ.rosario.18.01.2015.04

Sanabria, S. (2008). El papel del transporte en el crecimiento económico colombiano en la segunda mitad del siglo XX. XXVII-No. 46, XVII, 141-182.

SCT. (2016). Manual para obtener los Volúmenes de tránsito en carreteras. México: Secretaría de Comunicaciones y Transportes. Recuperado de https://www.sct.gob.mx/fileadmin/DireccionesGrales/DGST/Manuales/manual_volumen_de _transito/Manual_volumenes_2016_v2.pdf

Sun, L. (2016). LCCA-based design method for asphalt pavement. In Structural Behavior of Asphalt Pavements (pp. 549-600). Elsevier. https://doi.org/10.1016/B978-0-12-8499085.00008-0

Zamora, A. y Pedraza, O. (2013). El transporte internacional como factor de competitividad en el comercio exterior. Journal of Economics Finance and Administrative Science, 18(35), 108118. https://doi.org/10.1016/S2077-1886(13)70035-0 


\section{SEMBLANZA DE AUTORES}
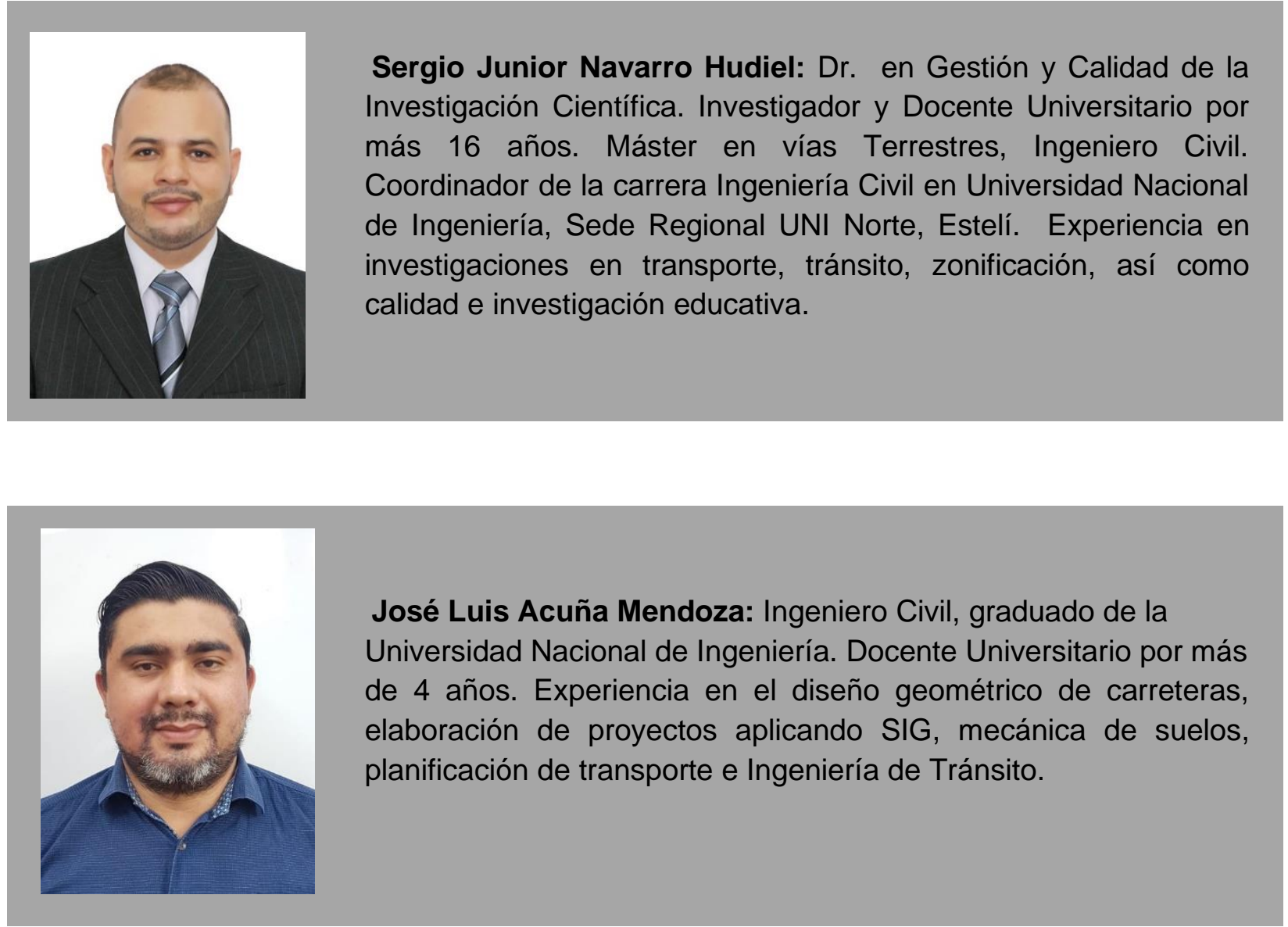